\title{
A Novel Ultrasensitive Gas Detector System Based on Carbon Nanotube Mixtures and Antenna Radiation Variation
}

\author{
Trang T. Thai ${ }^{(1)}$, Amil Haque ${ }^{(1)}$, Gerald R. DeJean ${ }^{(2)}$, and Manos M. Tentzeris ${ }^{(1)}$ \\ (1) School of ECE, Georgia Institute of Technology, Atlanta, GA 30332, USA \\ (2) Microsoft Research, One Microsoft Way, Redmond, WA 98052, USA \\ E-mail: trang.thai@gatech.edu
}

\begin{abstract}
A highly sensitive remote-sensing detector based on a mixture of double-walled carbon nanotubes has been designed to selectively detect different gases. The sensor consists of an antenna and a gas sensitive transmission line, operating at $60 \mathrm{GHz}$. The detection principle is based on different shifts of amplitude and phase of the antenna-feeding signal due to the presence of different gases, resulting in the change of the polarization and radiation pattern of the sensor antenna.
\end{abstract}

\section{Introduction}

In the current gas sensing technology, most sensor devices require some form of direct physical contact. Carbon nanotubes (CNTs) have been found to have many distinct properties that may enable the realization of the next generation of sensors with a very high sensitivity up to $1 \mathrm{ppb}$ (part per billion), an improvement of 2-3 orders of magnitude. Most CNT-based active sensors employ the change in resistance (easily affected by moisture), thermoelectric power, electrical breakdown voltage, or dielectric properties of the nanotubes for detection. Many of these sensors require a hightemperature operating conditions and direct physical contact that limit them from sensing the remote sites. Due to strong demands for monitoring hazardous gases in remote or rugged environments, integration of the sensors with wireless monitoring technology is desired for real-time remote sensing.

Recently, Chopra et al. demonstrated a CNT-coated resonator-based gas sensor that enables remote sensing [1]. The operation is based on the change of the effective relative permittivity of the resonator which results in a resonant frequency shift of 1-4 MHz when exposed to different gases including $\mathrm{NH} 3$ and $\mathrm{CO}$ at several hundreds ppb to several ppm (part per million) for a center resonant frequency of about $3.889 \mathrm{GHz}$. A coplanar waveguide $(\mathrm{CPW})$ transmission line filled with a mixture of double-walled carbon nanotubes (DWNTs) was observed by Dragoman et al. to show large differences in the modulus and phase of the signals before and after 15 hours of exposure the transmission line being exposed to nitrogen gas having low pressure of 5 bar [2][3]. Dragoman et al. reported a $2 \mathrm{~dB}$ difference in transmission modulus and $25^{\circ}$ phase shift at $60 \mathrm{GHz}$ for the $\mathrm{CNT}$ filled $\mathrm{CPW}$ line. In this paper, we advance this observation further and demonstrate an entirely novel design based on the same CNT mixture to selectively sense various gases. The sensor design employs the change in dielectric constant and the conductivity of the CNT mixture filled in the antenna/sensor substrate to alter the radiation pattern and axial ratio for easy detection remotely. 
Based on the results reported in [2][3], calculations performed in Agilent Design Systems and simulations in HFSS were performed to estimate the permittivity and loss tangent of the DWNT filled substrate. Due to the high loss of CNT based substrate, the signals at the end of the sensing transmission line need to be amplified before reaching the feed lines of the antenna. The performance of these antennas is simulated in Microstripes 7.5 by Flomerics Inc. to demonstrate the detection capability of the proposed sensor system. Although the DWNT CPW line in [2] was exposed to gas in long duration, from [4] it can be deduced that the response time of the CNT composites/mixtures can be within minutes in which the electrical properties of the CNT material is altered. Therefore the sensing system proposed in this paper has the potential of ultrasensitive real-time detection.

\section{Principles of Operation}

The hereby introduced sensor system is illustrated in the block diagram of Fig. 1. The transmission line is the sensing element in this system and is based on the CPW demonstrated in [3]. At $60 \mathrm{GHz}$, the CPW line gives the value of about $-9.5 \mathrm{~dB}$ unexposed to gas and gives a value close to $-7.5 \mathrm{~dB}$ when exposed to gas (nitrogen) with a phase shift of 250 [2]. The amplifier then adjusts the signals' power to the appropriate level of power to drive an antenna for detection performance.

The permittivity of the DWNT mixture without the presence of gas is estimated to be 2.85 , and the loss tangent at $60 \mathrm{GHz}$ is estimated to be 0.765 . After exposed to gas, the permittivity and the loss tangent at $60 \mathrm{GHz}$ of the DWNT mixture are estimated to be 1.90 and 0.698 , respectively. These parameters are applied to the transmission lines to obtain its output at $60 \mathrm{GHz}$.

\section{Simulation results}

The simulations performed in this section are based on the signals obtained after the amplifier stage shown in Fig. 1, in which the phase delay and the relative decrease in power are reserved and fed into the input port of the antennas. To simulate the conditions in the absence of gas, both ports are driven with a voltage of $1 \mathrm{~V}$ and 00 in phase; in the presence of gas conditions, one of these ports is driven with a voltage of $0.7 \mathrm{~V}$ and 250 delay in phase. This condition corresponds to the modulus and phase change reported by Dragoman et al. in the S21 response that was normalized by the amplifier. The antenna is designed on a double copper-clad Rogers Duroid RT5880 substrate and is operated at 60 GHz. The antenna topology is shown in Fig. 2. The benchmarking antenna-sensor is a regular patch antenna being fed by two microstrip lines (one "sensing" and one "control/insensitive to gas") that are placed $90^{\circ}$ from each other to initially (no present gas) produce circular polarization. The patch has the size of $1600 \times 1600 \mathrm{um}^{2}$. This topology generates two radiation lobes that are symmetric under regular conditions in which no gas is present to affect the sensing transmission line. As the signal on the "sensing" transmission line is altered, the signal being fed into one of the microstrip lines is altered accordingly with respect to the controlled transmission line. 
The signals coming from the sensing transmission line having its amplitude decreased by about $2 \mathrm{~dB}$ and its phase delay by 250 compared to the signals coming from the controlled line. Hence, the symmetry of radiation is destroyed due to unbalance in the feeding mechanism. The results are shown in Fig. 3 and 4. Notice the measurement results reported in [2] are applied directly to the change implemented at the ports here.

The second antenna is a regular patch antenna being fed by two microstrip lines that are placed 900 from each other. This topology generates two radiation lobes that are symmetric under regular condition in which no gas is present to affect the sensing transmission line. As the signal on the sensing transmission line is altered, the signal being fed into one of the microstrip line is altered accordingly with respect to the controlled transmission line, thus causing the unbalance in the feeding mechanism. This effect results in an asymmetric radiation lobes, thus a significant change in the radiation pattern occurs (Fig. 3 and 4). Therefore, modification in the axial ratio and radiation pattern gives a direct estimate of the type and the quantity of the detected gas.

\section{Conclusion}

The sensor system based on DWNTs was demonstrated to have high sensitivity could potentially be employed to selectively sense different gases such as ammonia, carbon dioxide, etc. This gas sensor system is miniaturized and can be implemented into any portable device related to human health, medical, and defense systems. The drawback of this type of sensor is the long recovery time at room temperature, however it can be easily incorporated into existing wireless network to perform remote sensing. This feature allows a unique sensing platform that conventional sensors, such as semiconductor or metal oxide sensors, lack.

\section{References}

[1] S. Chopra, A.Pham, J. Gaillard, A. Parker, A. M. Rao, "Carbon-nanotube-based resonant-circuit sensor for ammonia", Appl. Phys. Lett. 80, 4632 (2002).J.N.

[2] M. Dragoman, K. Grenier, D. Dubuc, L. Bary, R. Plana, E. Fourn, E. Flahaut, "Millimeter wave carbon nanotube gas sensor," App. Phy. Lett. 101, 106103 (2007)

[3] M. Dragoman, K. Grenier, D. Dubuc, L, Bary, E. Fourn, and R. Plana, “ Experimental determination of microwave attenuation and electrical permittivity of double-walled carbon nanotubes," App. Phys. Lett. 88, 153108 (2006)

[4] K. G. Ong, K. Zeng, C. A. Grimes, "A wireless, passive carbon nanotube-based gas sensor", IEEE Sens. J., 2, pp 82-88 (2002) S. Banba and H. Ogawa, "Small-sized MMIC amplifiers using thin dielectric layers," IEEE Trans, Microwave Theory Tech., Vol. 53, pp. 485-492, March 1995. 


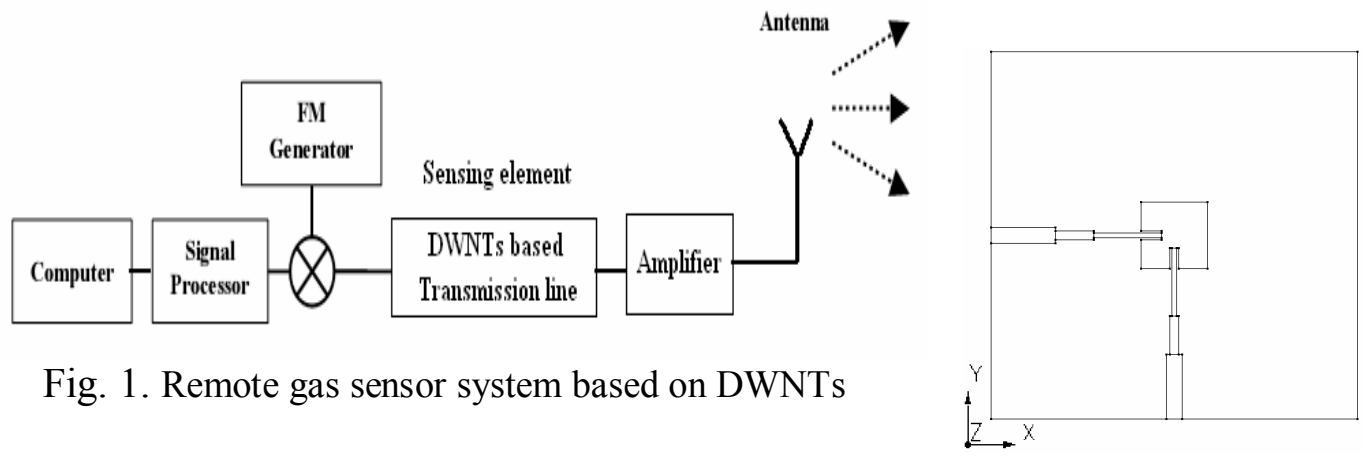

Fig. 2. The antenna

topology

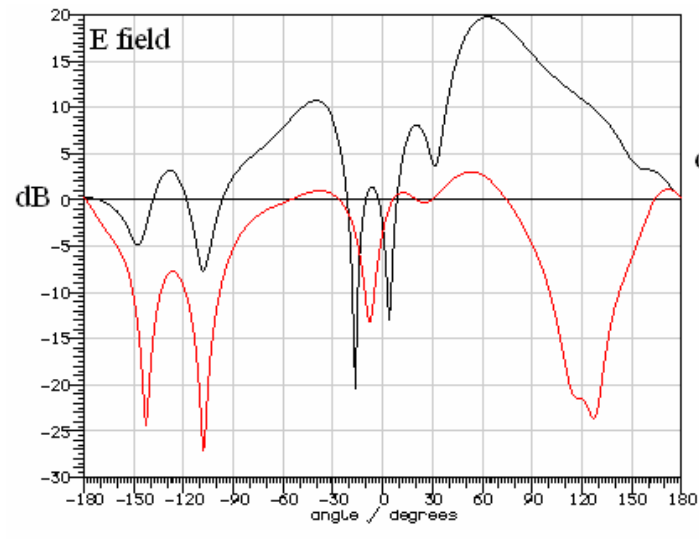

a) Before exposed to gas

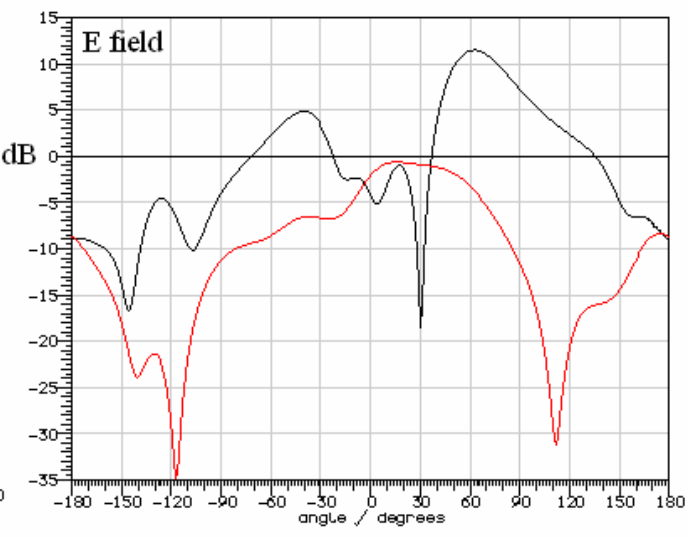

b) After exposed to gas

\section{- Co-polarization \\ - Cross polarization}

Fig .3. The E-field captured on the E-plane for both co-polarization and crosspolarization

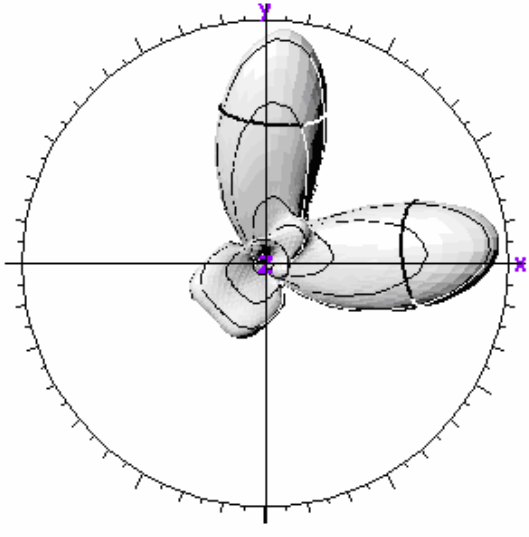

a) Before exposure to gas

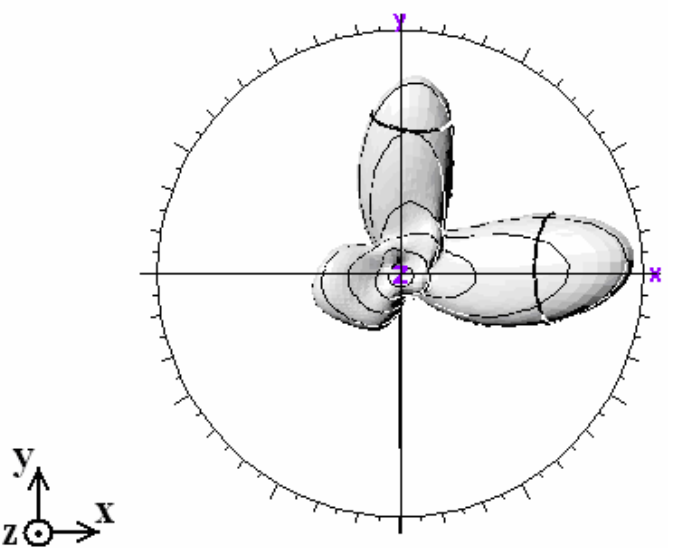

b) After exposure to gas

Fig. 4. The 3-dimensional radiation pattern of the antenna sensing system 